\title{
QoS Performance Analysis of MANET with Bandwidth Estimation
}

\author{
Prem Chand \\ Research Scholar \\ Department of CSE, MRIU \\ Faridabad, Haryana, India
}

\author{
M.K.Soni \\ E.D. \& Dean \\ FET, MRIU, \\ Faridabad, Haryana, India.
}

\begin{abstract}
Mobile Ad-hoc network (MANET) has become an important edge network to provide urgent situation access to remote areas and in a metropolitan scale. These applications require Quality of Service (QoS) parameters such as: bandwidth, throughput, hop-count and energy to be adequate so that a reliable connection between participating nodes is maintained. This paper is an effort to study the problem of nodes having bandwidth constraint. To study the effect of same a simulator is designed in MATLAB-7.0. Simulation results show that the number of hop-counts decreases as we increase the percentage of low bandwidth nodes in the network, it was also concluded that the throughput rate decreases as we increases the number of low bandwidth nodes in the network.
\end{abstract}

\section{General Terms}

In this paper a simulator in MATLAB has been designed for shortest path finding and throughput calculation using Dijkstra's algorithm. This paper is an effort to study performance of MANET with low bandwidth nodes.

\section{Keywords}

Ad-hoc networks, Bandwidth, Throughput, Hop-count, Performance.

\section{INTRODUCTION}

Mobile Ad-hoc Network (MANET) [1] is an autonomous network that can be formed without any established infrastructure. Mobile hosts communicate with each other using multi-hop [2] wireless links. Each node in the network also acts as a router. It is the responsibility of each node to forward data packets for other nodes. As these networks are rapidly deployable and they don't rely on external infracture, it makes them an ideal candidate for many applications. Due to the quick and economically less demanding deployment MANET find applications in several areas. Some of them are: Collaborative and distributed computing, emergency operations and military applications. Along with a good range of applications there are many issues that give a significant impact on the performance [3] of mobile ad-hoc networks (MANETs), Routing, Multicasting, Self Organization, Security, Energy Management and Quality of Service are some of them. The issue of Quality of Service [4] has been taken as the main consideration throughout the paper. QoS is the performance level of services offered by a service provider or a network to the user. Quality of Service depends upon a few QoS parameters [5]: As different applications have different requirements, their level of QoS and associated QoS parameters also differ from application to application. For example, for multimedia applications the bandwidth and delay are key parameters. In this paper we carry out a systematic performance study of the two QoS factors on the basis of varying percentage of low bandwidth [6] nodes in the network. We have used the means of simulation using MATLAB (7.0), a simulator is being designed in MATLAB 7.0. It gathers data about number of hop-count [7] (number of nodes between source and destination for successful routes) and throughput rate [8] (total number of packets received by the destination to the total number of packets send by source). The simulator uses dijkstra algorithm to implement shortest path routing. To evaluate the efficacy of MANET for nodes having lower bandwidth, the nodes were made unreachable by assigning low bandwidth [9] to a group of some specified percentage nodes in the network. This work is ordered as follows. We described the related work in section 2 and simulation model and results in Section 3. Section 4 concludes the paper along with future scope.

\section{RELATED WORK IN MANET PROTOCOLS}

The key issue [10] with ad-hoc networking is how to send a message from one node to another with no direct link. The nodes in the network are moving around randomly, and it is very difficult that which nodes are directly linked together. Same time topology of the network is constantly changing and it is very difficult for routing process. Another key issue is the problem of bandwidth allocation in wireless networks. Even if the progress is being made for high-speed wireless communications, such as the introduction of $3 \mathrm{G}$ and WLAN, bandwidth is still the major bottleneck in wireless networks due to the physical limitation of wireless media [11]. Bandwidth estimation is a basic function that is required to provide QoS in MANETs [12]. It is a way to determine the data rate available on a network route. It is of interest to users wishing to optimize end-to-end transport performance, overlay network routing, and peer-to-peer file distribution. A number of policies had been proposed [13] for optimized bandwidth estimation some of them are taken here for discussion purpose.

\subsection{Dynamic Bandwidth Allocation Algorithm for MANET}

Dynamic bandwidth provisioning in QoS networks has recently attracted a lot of research attention due to its prospective to attain efficient resource utilization to network users. In [14], the authors proposed a method of Achieving Maximum Flow in Interference-Aware Wireless Sensor Networks with Smart Antennas. The core provisioning architecture consists of a set of lively node and algorithms for interior nodes and core nodes. The provisioning algorithm assume a self-adaptive mechanism to adjust service weights of weighted fair queuing schedulers at core routers and it reduces the boundary bandwidth while receiving a congestion signal 
from a node provisioning module and affords periodic bandwidth rearrangement.

\subsection{Intrusive Bandwidth Estimation}

Techniques: The intrusive approaches techniques are based on end-to-end probe [15] packets to estimate the available bandwidth along a path.

\subsection{Passive Bandwidth Estimation}

Techniques: The passive approaches techniques uses local information on the used bandwidth and that may exchange this information via local broadcasts [17].

\section{SIMULATION AND RESULTS}

We have used a detailed simulation model based on MATLAB 7.0, for system/protocol modeling. MATLAB is a high-performance language for technical computing. It integrates computation, visualization, and programming in an easy-to-use environment where problems and solutions are expressed in familiar mathematical notation. Typical uses include Math and computation Algorithm development Data acquisition Modeling, simulation, and prototyping Data analysis, exploration, and visualization Scientific and engineering graphics Application development, including graphical user interface building.

The result part has been divided into four different areas viz. Snapshot; Setup Parameters, Impact of low bandwidth nodes on hop-count and Impact of low bandwidth nodes on throughput.

3.1. Snapshot: The figures mentioned below are the various outcomes which came during the simulation running process.

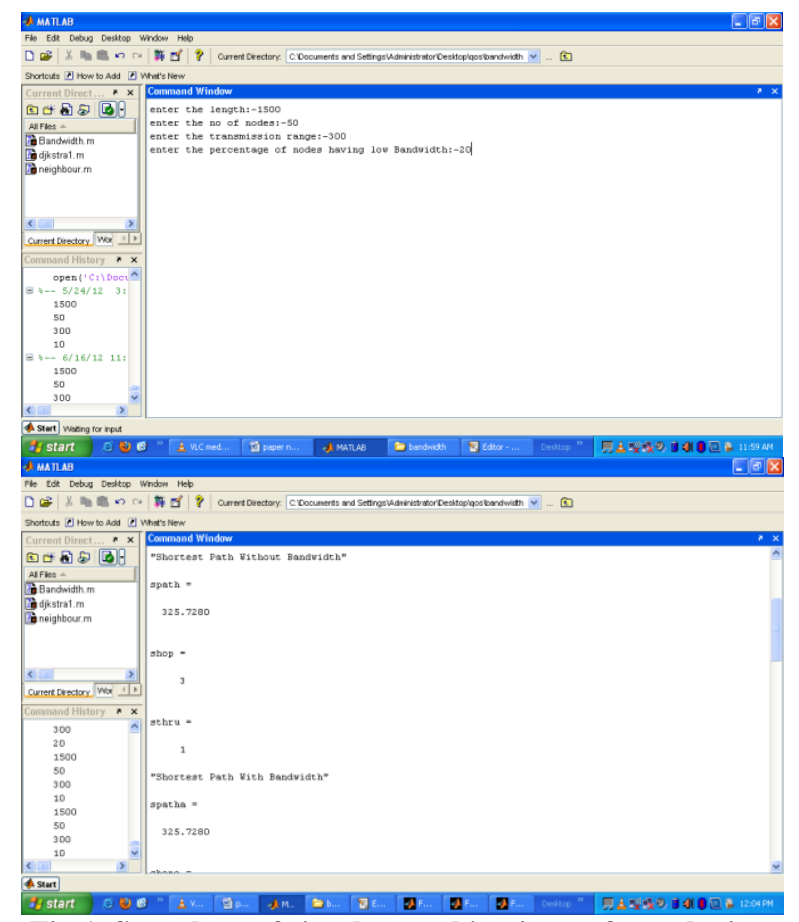

Fig 1. Snapshots of simulator taking input \& producing output.

Here Fig 1 shows the working model of simulator as it is taking input values in terms of length of network, number of nodes, percentage of low bandwidth nodes etc. Similarly Fig 2 as a outcome of simulator shows nodes $3,13,22,31$ and 46 as low bandwidth nodes.

3.2 Setup Parameters: We have primarily selected the number of hop-count and throughput rate under bandwidth constraint. Here hop-count is defined as the number of nodes between source and destination for successful routes and throughput rate is the total number of packets received by the destination to the total number of packets sends by source. The table given below gives the setup parameters for the developed simulator.

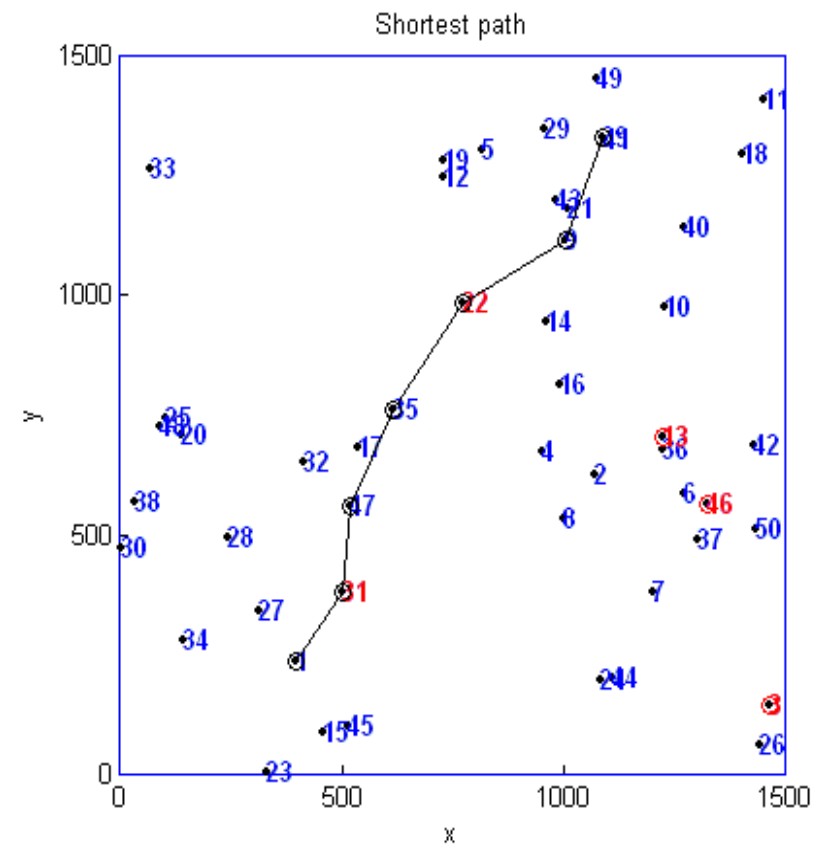

Fig 2. Number of hop-counts in a shortest path

Table1.

\begin{tabular}{|l|l|}
\hline Area & $1500 * 1500$ \\
\hline Number of Nodes & 50 \\
\hline Transmission Range & 300 \\
\hline $\begin{array}{l}\text { Percentage of nodes having } \\
\text { low bandwidth }\end{array}$ & $\begin{array}{l}\text { Varies from 0 to 100 } \\
\text { percentage (with a interval } \\
\text { of 10) }\end{array}$ \\
\hline
\end{tabular}

\subsection{Impact of Low bandwidth on hop-count:}

The Fig 3 shown below shows that as we gradually increases the percentage of low bandwidth nodes form $10 \%$ to $20 \%$ and keep on going up to $100 \%$, the hop-count decreases; It shows that the routes which required more intermediate nodes are not forming in the network. Simply longer routes were not being established with the growth of low bandwidth nodes.

\subsection{Impact of Low bandwidth on throughput:}

Throughput [18] of a network is the ratio of number of packets received by the destination to the total number of packets send by source. Here in this study as shown in Fig 4 , 
the throughput rate is going to decrease from level 1 to the level 0 in proportionate to increasing percentage [19] of low bandwidth nodes in the network and it reaches to level 0 with $100 \%$ low bandwidth nodes.

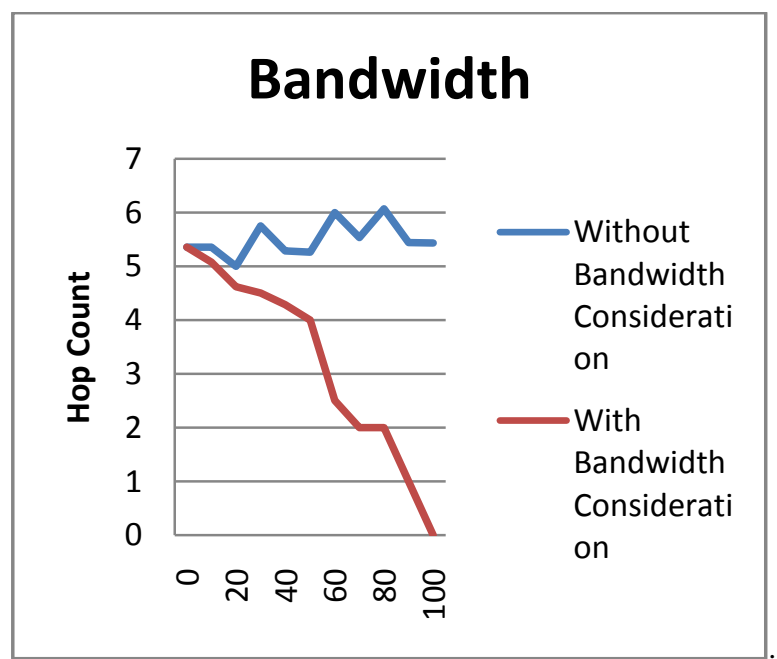

Fig 3. Effect of low bandwidth on hop count

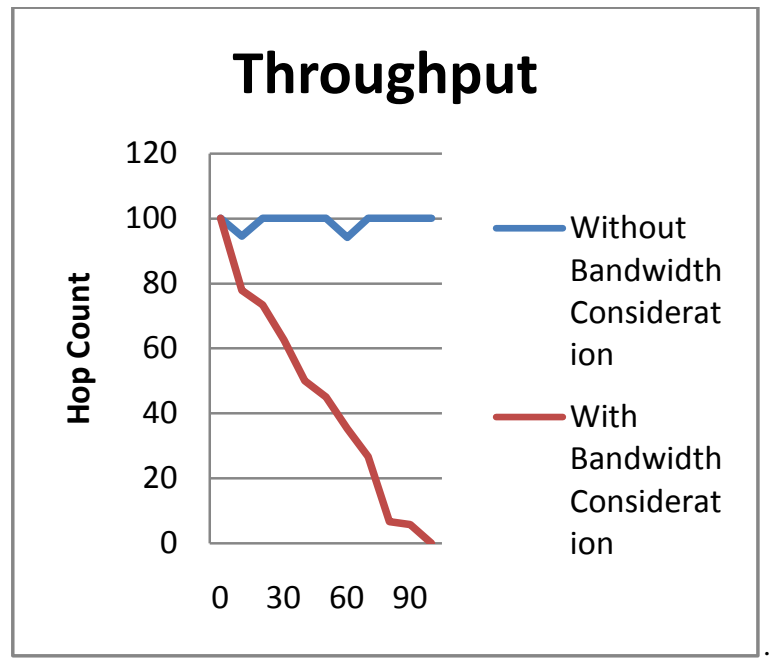

Fig 4. Effect on throughput of low bandwidth nodes

\section{CONCLUSION AND FUTURE SCOPE}

In this paper, we presented a simulator which is designed in MATLAB-7.0. The simulator uses dijkstra algorithm to implement shortest path routing. To evaluate the efficacy of MANET for nodes having lower bandwidth, the nodes were made unreachable by assigning a distinct bandwidth to distinct nodes. This paper is an effort to study systematic performance of two QoS factors (Hop count and Throughput) on the basis of varying percentage of low bandwidth nodes in the network. The simulator designed in MATLAB 7.0 gathers data about number of hop-count (number of nodes between source and destination for successful routes) and throughput rate (total number of packets received by the destination to the total number of packets send by source). This information later on is being analyzed with nodes having normal/ideal bandwidth, for which comparative graph have been discussed in the paper. In future, the scope for this paper can be found in designing routing protocols where bandwidth utilization of each node is required in advance.

\section{REFERENCES}

[1] M. Joa-Ng and I. T. Lu (1999): “A Peer-to-Peer ZoneBased Two-Level Link State Routing for Mobile Ad-hoc Networks". IEEE Journal on Selected Areas in Communications, vol. 17, no. 8, pp. 1415-1425.

[2] David A. Maltz, Josh Broch, Jorjeta Jetcheva, and David B.Johnson(1999): "The Effects of On-Demand Behavior in Routing Protocols for Multi-Hop Wireless Ad Hoc Networks". IEEE Journal on Selected Areas of Communications, 17(8):1439-1453.

[3] Per Johansson, Tony Larsson, Nicklas Hedman, Bartosz Mielczarek, and Mikael Degermark. Routing Protocols for Mobile Ad-hoc Networks-A Comparative Performance Analysis. In Proceedings of the Fifth International Conference on Mobile Computing and Networking (MobiCom'99). ACM, August 1999.

[4] L. Chen and W.B. Heinzelman, "QoS-Aware Routing Based on Bandwidth Estimation for Mobile Ad Hoc Networks," IEEE J. Selected Areas in Comm., vol. 23, no. 3, pp. 561-572, Mar. 2005.

[5] Perkins, D., Hughes, H., Owens, C., 2002, "Factors effecting the Performance of Ad hoc Networks," IEEE International Conference on Communications, IEEE Press, 2048-2052.

[6] Chen, L., Heinzelman, W.B., 2005, "QoS-aware Routing based on Bandwidth Estimation for Mobile Ad-hoc Networks" IEEE Journal on Selected Areas in Communications, Volume 23, Issue 3, March, 561-572.

[7] C. C. Chiang, H. K. Wu, W. Liu and M. Gerla(1997): "Routing in Clustered Multi-Hop Mobile Wireless Networks with Fading Channel". Proceedings of IEEE SICON 1997, pp. 197-211.

[8] Mayhew, G.L., 2007, "Quality of Service in Mission Orientated Ad-hoc Networks", IEEE Aerospace Conference, 3-10 March, 1-9.

[9]. Ziane, S., Mellouk, A., 2007, “AMDR: A Reinforcement Adaptive Mean Delay Routing Algorithm for MANET", IEEE Global Telecommunications Conference, 26-30 November, 726-730.

[10]. Canales, M., Gallego, J.R., Hernandez-Solana, A., Valdovinos, A., 2006, "Cross-Layer Routing for QoS Provision in Multiservice Mobile Ad Hoc Networks", IEEE 17th International Symposium on Personal, Indoor and Mobile Radio Communications, September, 1-5.

[11]. de Renesse, R., Friderikos, V., Aghvami, H., 2007, "Cross-layer Cooperation for Accurate Admission Control Decisions in Mobile Ad hoc Networks", IET Communications, Volume 1, Issue 4, August, 577-586.

[12]. Romdhani, L., Bonnet, C., 2005, "A Cross-layer Ondemand Routing Protocol for Delay-Sensitive Applications", IEEE 16th International Symposium on Personal, Indoor and Mobile Radio Communications, Volume 2, 11-14 September, 994-998.

[13]. Abdullah, J., Parish, D., 2007, "Impact of QoS Routing Metrics for MANETs in the Pervasive Computing Environment"; International Conference on Wireless and Optical Communications Networks 2007, 2-4 July, 1-5. 
[14] X. Huang, J.Wang, Y. Fang, “Achieving Maximum Flow in Interference-Aware Wireless Sensor Networks with Smart Antennas", Elsevier Journal on Ad hoc Networks, vol. 5, pp. 885-896, February 2007.

[15] I. Sheriff, E.M.B. Royer, "Multipath Selection in MultiRadio Mesh Networks", Proceedings of $3^{\text {rd }}$ International Conference on Broadband Communications (BROADNETS), pp. 1-11, October 2006.

[16] J.W. Tsai, T. Moors, "Interference-Aware Multipath Selection for Reliable Routing in Wireless Mesh Networks", Proceedings of 3rd International Conference on Mobile Ad hoc and Sensor Systems (MASS), pp. 1-6, October 2007.
[17] J.Y. Teo, Y. Ha, C.K. Tham, "Interference-Minimized Multipath Routing with Congestion Control in Wireless Sensor Network for High-Rate Streaming", IEEETransactions on Mobile Computing, vol. 7, no. 9, pp.1124-1137, September 2008.

[18] R.D. Haan, R.J. Boucherie, J.K.V. Ommeren, "The Impact of Interference on Optimal Multi-path Routing in Ad Hoc Networks", Proceedings of 20th International Teletraffic Congress (ITC), LNCS 4516, pp. 803-815, June 2007

[19] Kone, V., Nandi, S., 2006, "QoS Constrained Adaptive Routing Protocol for Mobile Adhoc Networks", $9^{\text {th }}$ International Conference on Information Technology, 18-21 December, 40-45. 